\title{
Searches for lepton flavour violation at a linear collider
}

\section{E. Carquín, ${ }^{a}$ J. Ellis, ${ }^{b, c}$ M.E. Gómez ${ }^{d}$ and S. Lola ${ }^{b, e}$}

\author{
${ }^{a}$ Instituto de Estudios Avanzados en Ciencias e Ingeniera y \\ Centro Cientfico-Tecnolgico de Valparaso, Physics Department, \\ Technical University Federico Santa Mara, \\ Avenida de Espaa 1680, Valparaso, Chile \\ ${ }^{b}$ Theory Division, Physics Department, CERN, \\ CH-1211 Geneva 23, Switzerland \\ ${ }^{c}$ Theoretical Particle Physics and Cosmology Group, Department of Physics, \\ King's College London, \\ Strand, London WC2R 2LS, U.K. \\ ${ }^{d}$ Department of Applied Physics, University of Huelva, \\ Avenida 3 de Marzo, 21071 Huelva, Spain \\ ${ }^{e}$ Department of Physics, University of Patras, \\ Panepistimioupoli Rio, GR-265 00 Patras, Greece \\ E-mail: edson.carquin@usm.cl, john.ellis@cern.ch, \\ mario.gomez@dfa.uhu.es, magda.lola@cern.ch
}

ABSTRACT: We investigate the prospects for detection of lepton flavour violation in sparticle production and decays at a Linear Collider (LC), in models guided by neutrino oscillation data. We consider both slepton pair production and sleptons arising from the cascade decays of non-leptonic sparticles. We study the expected signals when leptonflavour-violating (LFV) interactions are induced by renormalization effects in the Constrained Minimal Supersymmetric extension of the Standard Model (CMSSM), focusing on the subset of the supersymmetric parameter space that also leads to cosmologically interesting values of the relic neutralino LSP density. Emphasis is given to the complementarity between the LC, which is sensitive to mixing in both the left and right slepton sectors, and the LHC, which is sensitive primarily to mixing in the right sector. We also emphasize the complementarity between searches for rare LFV processes at the LC and in low-energy experiments.

KEYWORDS: Supersymmetry Phenomenology

ARXIV EPRINT: 1106.4903 


\section{Contents}

1 Introduction $\quad 1$

2 LFV in slepton pair production $\quad 2$

2.1 CMSSM with LFV 2

2.2 LFV cross sections 3

3 Supersymmetric parameter space $\quad 5$

4 Opportunities for LFV observation at the LC 9

5 Study of LFV at the LC 12

6 Conclusions 17

\section{Introduction}

In recent years, a plethora of data from atmospheric [1], solar [2-4] and long-baseline reactor $[5,6]$ and accelerator $[7,8]$ neutrino experiments have established the existence of neutrino masses and oscillations with near-maximal $\nu_{\mu}-\nu_{\tau}$ and large $\nu_{e} \rightarrow \nu_{\mu}$ mixing. A natural expectation in this context is that charged-lepton-flavour violation (LFV) should occur at some level. This may be enhanced sufficiently to become observable in a class of theories predicting new physics at the $\mathrm{TeV}$ scale accessible to colliders, particularly in supersymmetric theories. There are several sources of lepton flavour violation in such theories, which could have unacceptably large LFV if the soft supersymmetry-breaking masses of different sfermion flavours were not universal at some level. For this reason, it is often assumed that these masses are equal at the grand-unification scale, as in the Constrained Minimal Supersymmetric extension of the Standard Model (CMSSM).

Even if the sfermion mass matrices are diagonal at the unification scale, as in the CMSSM, quantum corrections would modify this structure while running from the GUT scale to low energies. This effect is particularly interesting in see-saw models for neutrino masses, where the Dirac neutrino Yukawa couplings cannot be diagonalised simultaneously with the charged-lepton and slepton mass matrices [9]. Given the large mixing of the corresponding neutrino species, charged-lepton-flavour violation may occur at enhanced rates for sufficiently small soft supersymmetry-breaking masses, giving rise to observable signals such as $\mu \rightarrow e \gamma, \mu-e$ conversion, $\tau \rightarrow \mu \gamma$ and $\tau \rightarrow e \gamma$ [10-24].

Other charged-lepton-flavour violating possibilities that have been considered include slepton pair production at a Linear Collider (LC) [25-30], and also signals at the LHC [31-37], particularly in $\chi_{2}^{0} \rightarrow \chi+e^{ \pm} \mu^{\mp} \chi_{2}^{0} \rightarrow \chi+\mu^{ \pm} \tau^{\mp}$ decays (here $\chi$ is the lightest neutralino, assumed to be the lightest supersymmetric particle (LSP), and $\chi_{2}^{0}$ is 
the second-lightest neutralino). These decays potentially provide search prospects that are complementary to direct searches for the flavour-violating decays of charged leptons. However, most of the previous studies of LFV at a LC use a low-mass spectrum (focusing on $P_{c m}<400 \mathrm{GeV}$ or $\sqrt{s}<800 \mathrm{GeV}$ ) and the points chosen for study do not always satisfy all the relevant phenomenological and experimental bounds (e.g., [38]), which are now being improved by the LHC [39-42]. Moreover, previous analyses have not always taken into account the constraints on the cosmological relic LSP density imposed by WMAP and other experiments [43].

In the current paper, we revisit the various detection channels for lepton flavour violation at a Linear Collider, studying the complementarity between different processes. In all cases, we focus on regions that satisfy not only all phenomenological constraints, but also the cosmological relic density considerations. We extend previous results to somewhat heavier sparticle spectra, and make comparisons with the expectations for experimental sensitivity at the LHC.

Our paper is structured as follows. In section 2, we briefly review the theoretical framework and sources of slepton mixing and summarize the possibilities for observable signatures in slepton production at a LC. In section 3, we discuss the relevant supersymmetric parameter space, and pick representative points motivated by both phenomenological and cosmological considerations. In section 4 , we look at the expected slepton mixing parameters, with emphasis on the region where LC experiments could give information additional to that obtainable from low-energy LFV decays. In section 5, we discuss in more detail the cross sections, comparing with the expected signals at the LHC, where appropriate. Finally, in section 6 , we summarize our conclusions and outlook.

\section{LFV in slepton pair production}

\subsection{CMSSM with LFV}

In the unrotated charged-lepton flavour basis $\tilde{\ell}_{i}=\left(\tilde{e}_{L}, \tilde{\mu}_{L}, \tilde{\tau}_{L}, \tilde{e}_{R}^{*}, \tilde{\mu}_{R}^{*}, \tilde{\tau}_{R}^{*}\right)$, the charged slepton mass matrix is:

$$
M_{\tilde{\ell}}^{2}=\left(\begin{array}{cc}
M_{\mathrm{LL}}^{2} & M_{\mathrm{LR}}^{2} \\
M_{\mathrm{RL}}^{2} & M_{\mathrm{RR}}^{2}
\end{array}\right),
$$

where

$$
\begin{aligned}
& M_{\mathrm{LL}}^{2}=m_{\ell}^{\dagger} m_{\ell}+M_{L}^{2}-\frac{1}{2}\left(2 m_{W}^{2}-m_{Z}^{2}\right) \cos 2 \beta I, \\
& M_{\mathrm{RR}}^{2}=m_{\ell}^{\dagger} m_{\ell}+M_{R}^{2}-\left(m_{Z}^{2}-m_{W}^{2}\right) \cos 2 \beta I, \\
& M_{\mathrm{LR}}^{2}=\left(A^{e}-\mu \tan \beta\right) m_{\ell}, \\
& M_{\mathrm{RL}}^{2}=\left(M_{\mathrm{LR}}^{2}\right)^{\dagger} .
\end{aligned}
$$

Here we parametrize trilinear soft supersymmetry-breaking terms as $A_{i j}^{e}\left(\lambda_{e}\right)_{i j}$, where the $\lambda_{e}$ are the respective Yukawa couplings. For universal soft terms at some high input scale, one has

$$
M_{L}^{2}=M_{R}^{2}=m_{0}^{2} I, \quad A_{i j}^{e}=A_{0} \delta_{i j},
$$


whereas flavour-mixing entries may be parametrized by:

$$
\delta_{X X}^{i j}=\left(M_{X X}^{2}\right)^{i j} /\left(M_{X X}^{2}\right)^{i i} \quad(X=L, R) .
$$

The correspondence between the mixing parameters $\delta_{X X}$ and the flavour mixing parameters used in the phenomenological study of slepton production cross sections is easily derived. For example, defining the splitting of the third-generation soft supersymmetry-breaking mass terms as might be generated by the renormalization-group equations (RGEs) by

$$
K_{X X}=1-\left(M_{X X}^{2}\right)^{33} /\left(M_{X X}^{2}\right)^{22},
$$

the slepton mixing angle $\tilde{\theta}_{23}$ and the splitting of the second- and third-generation mass eigenstates $\Delta \tilde{m}_{23}$ can be written as:

$$
\begin{aligned}
\tan \left(2 \tilde{\theta}_{23}\right) & =2 \delta_{X X} / K_{X X} \\
\Delta \tilde{m}_{23} & =\frac{\left(M_{X X}^{2}\right)^{22}}{\tilde{m}} \frac{\delta_{X X}}{\sin \left(2 \tilde{\theta}_{23}\right)}
\end{aligned}
$$

where $\tilde{m}=\frac{1}{2}\left(m_{2}+m_{3}\right)$.

The evaluation of the LFV observables is done by performing the diagonalization of the slepton mass matrices (see [11], for instance), inserting the full rotation matrices in the lepton-slepton-gaugino vertices and summing over all the mass eigenstates of the exchanged particles.

\subsection{LFV cross sections}

As already discussed in the introduction, charged-lepton flavour violation at a LC may occur either directly in slepton pair production or indirectly via slepton production in cascade decays $[25,26]$. Processes leading to lepton production in the decays of a pair of sleptons include

$$
\begin{aligned}
& e^{+} e^{-} \rightarrow \tilde{\ell}_{i}^{-} \tilde{\ell}_{j}^{+} \rightarrow \tau^{ \pm} \mu^{\mp} \tilde{\chi}_{1}^{0} \tilde{\chi}_{1}^{0}, \\
& e^{+} e^{-} \rightarrow \tilde{\nu}_{i} \tilde{\nu}_{j}^{c} \rightarrow \tau^{ \pm} \mu^{\mp} \tilde{\chi}_{1}^{+} \tilde{\chi}_{1}^{-},
\end{aligned}
$$

for which representative Feynman diagrams are shown in figure 1. Slepton production may also result from the the cascade decays of the heavier gauginos, e.g., via the processes

$$
\begin{aligned}
& e^{+} e^{-} \rightarrow \tilde{\chi}_{2}^{ \pm} \tilde{\chi}_{1}^{\mp} \rightarrow \tau^{ \pm} \mu^{\mp} \tilde{\chi}_{1}^{+} \tilde{\chi}_{1}^{-}, \\
& e^{+} e^{-} \rightarrow \tilde{\chi}_{2}^{0} \tilde{\chi}_{1}^{0} \rightarrow \tau^{ \pm} \mu^{\mp} \tilde{\chi}_{1}^{0} \tilde{\chi}_{1}^{0} .
\end{aligned}
$$

These amplitudes differ in the interference terms, and read as follows:

$$
\begin{aligned}
M_{\alpha \beta}^{\text {pair }} & =\sum_{i} M_{P}^{\text {pair }} \frac{i}{q^{2}-\tilde{m}_{i}^{2}+i \tilde{m}_{i} \Gamma_{i}} T_{i \alpha} M_{D}^{+} \frac{i}{p^{2}-\tilde{m}_{i}^{2}+i \tilde{m}_{i} \Gamma_{i}} T_{i \beta}^{*} M_{D}^{-}, \\
M_{\alpha \beta}^{\text {casc }} & =\sum_{i} M_{P}^{\text {casc }} T_{i \alpha} \frac{i}{q^{2}-\tilde{m}_{i}^{2}+i \tilde{m}_{i} \Gamma_{i}} T_{i \beta}^{*} M_{D}^{-},
\end{aligned}
$$



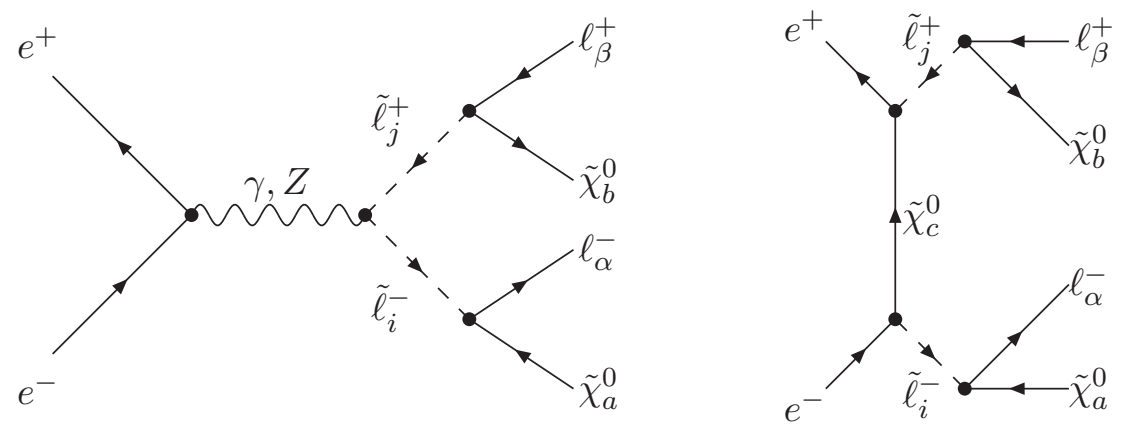

Figure 1. Feynman diagrams for $e^{+} e^{-} \rightarrow \tilde{\ell}_{j}^{+} \tilde{\ell}_{i}^{-} \rightarrow \ell_{\beta}^{+} \ell_{\alpha}^{-} \tilde{\chi}_{b}^{0} \tilde{\chi}_{a}^{0}$. The arrows on scalar lines indicate the flow of lepton number. Similar diagrams - appropriately modified — exist for charginos.

for pair production and cascade slepton production, respectively, where $M_{P}$ and $M_{D}$ are the production and decay amplitudes for sleptons in the absence of LFV, and $T_{i \alpha}$ parametrizes the lepton-flavour-mixing matrix element.

Complete expressions for the respective cross sections are given in [27], and used in our work. However, intuition may be developed by first looking at the simplified case including only 2-3 slepton mixing. In this case, the only diagrams contributing to the cross sections are those mediated by $\gamma$ and $Z^{0}$ exchange. Even simpler formulae can be obtained in certain limits, such as the cases of narrow widths and small mass differences between the sleptons of different generations. For example, if $\Delta \tilde{m}_{i j} \ll \tilde{m}=\frac{1}{2}\left(m_{2}+m_{3}\right)$ and $\tilde{m} \bar{\Gamma}_{i j} \simeq\left(\tilde{m}_{i} \Gamma_{i}+\tilde{m}_{j} \Gamma_{j}\right) / 2 \ll \tilde{m}^{2}$, in the presence of $2-3$ mixing the cross sections for pair and cascade slepton production are given by [44]:

$$
\begin{aligned}
& \sigma^{\text {pair }}=\chi_{23}\left(3-4 \chi_{23}\right) \sin ^{2} 2 \tilde{\theta}_{23} \sigma\left(\bar{f} f \rightarrow \tilde{\ell}^{+} \tilde{\ell}^{-}\right) B r\left(\tilde{\ell}^{+} \rightarrow \ell^{+} X\right) B r\left(\tilde{\ell}^{-} \rightarrow \ell^{-} Y\right), \\
& \sigma_{\alpha \beta}^{\text {casc }}=\chi_{23} \sin ^{2} 2 \tilde{\theta}_{23} \sigma\left(f f^{\prime} \rightarrow \ell^{+} X \tilde{\ell}^{-}\right) B r\left(\tilde{\ell}^{-} \rightarrow \ell^{-} Y\right),
\end{aligned}
$$

where $X, Y$ represent gauginos in the final state, and $\sigma\left(\bar{f} f \rightarrow \tilde{\ell}^{+} \tilde{\ell}^{-}\right)$and $\operatorname{Br}\left(\tilde{\ell}^{ \pm} \rightarrow \ell^{ \pm} X\right)$ are the cross sections and branching ratios in the absence of flavour violation. In the case of flavour conserving cascade decays, $\sigma\left(f f^{\prime} \rightarrow \ell^{+} X \tilde{\ell}^{-}\right)$includes the intermediate production of two gauginos and the subsequent decay of the heavier into a lepton-slepton pair. LFV enters via $\chi_{23} \equiv x_{23}^{2} / 2\left(1+x_{23}^{2}\right)$ where $x_{23} \equiv \Delta \tilde{m}_{23} / \bar{\Gamma}_{23}$, and $\sin ^{2} 2 \tilde{\theta}_{23}$ parametrizes the slepton mixing angle. In the limit $x_{23} \gg 1$, interference can be neglected and the results are even further simplified. However, in the case that the interference term dominates, it suppresses LFV significantly.

The exact experimental signatures of the above effects depend on the decay chains, as discussed in previous analyses [27]. Channels involving the lightest charginos are potentially interesting, particularly those with hadronic chargino decays, since the lightest neutralino is 'seen' as missing energy. In general, the dominant chargino decay is $\tilde{\chi}_{1}^{+} \rightarrow \tilde{l} \nu$, whereas channels such as $\tilde{\chi}_{1}^{+} \rightarrow q_{u} q_{d} \tilde{\chi}^{0}$ are relevant when $m_{\tilde{\chi}_{1}^{+}}<m_{\tilde{\tau}_{1}}$ or, in other words, when the $\tilde{\chi}_{1}^{+}$is the NLSP. Channels leading to hadronic $\tilde{\chi}_{1}^{+}$decays [27] are mediated only by left sleptons, whilst $e^{+} e^{-} \rightarrow \tau^{ \pm} \mu^{\mp}+2 \chi^{0}$ may be mediated by either right or left sleptons. However, points with $m_{\tilde{\chi}_{1}^{+}}<m_{\tilde{\tau}_{1}}$ are disfavored in the framework of the CMSSM. For instance, 
the previously proposed point with $\tan \beta=3, m_{0}=100 \mathrm{GeV}, M_{1 / 2}=200 \mathrm{GeV}$ [28] was excluded by the LEP $m_{h}$ bound [38] and is now incompatible also with LHC limits [39-41]. This could perhaps be achieved in the focus-point region, where $M_{1 / 2}<1 \mathrm{TeV}$ and $m_{0}>$ several $\mathrm{TeV}$, but slepton masses in this region are very heavy and hence LFV processes are more suppressed.

The channel $e^{+} e^{-} \rightarrow \tau^{ \pm} \mu^{\mp}+2 \chi^{0}$ was studied in [30], where it was found that a signal of $\sim 1 \mathrm{fb}$ could be distinguished from the background. Only slepton mixing in the left sector arising from see-saw neutrino mass models was included in [30], and the limit from $\tau \rightarrow \mu \gamma$ was at the time less restrictive. As shown in [37], reasonable rates of LFV processes at the LHC in the slepton sector are possible while preserving the bound on $\tau \rightarrow \mu \gamma$ if one assumes mixing also among the right sleptons, as may occur in non-minimal GUT theories.

\section{Supersymmetric parameter space}

From the above discussion, it is clear that the dependence on the supersymmetric parameter space is particularly crucial, since the chains of cascade decays that will dominate, as well as the relative strengths of the signals and backgrounds, depend on the mass hierarchies of the superpartners.

We start with some preliminary considerations of the relevant channels within the CMSSM parameter space. In previous work [37, 45] a region that is promising from the cosmological point of view was discussed, in which sizable $\tilde{\tau}-\chi$ coannihilations or directchannel $H / A$ resonances lead to values of $\Omega_{\chi} h^{2}$ consistent with WMAP. As a representative of the coannihilation case, we chose $\tan \beta=35$, a value that leads to a relatively heavy sparticle spectrum compatible with WMAP. At $\tan \beta=45$ we found points in the resonance funnel, which have larger scalar masses since $m_{0}$ can take higher values than in the coannihilation area. We did not consider the focus-point region of the CMSSM because the super-heavy slepton masses there lead to reduced LFV effects. We note that these regions are compatible with the LHC constraints on supersymmetry, which indicate that $M_{1 / 2}>450 \mathrm{GeV}$ and favour $\tan \beta>15$ [42].

The physical values of the masses are obtained by integrating the renormalization group equations from $M_{G U T}$ down to low energies. We use the two-loop supersymmetric renormalization group equations [46] except for the trilinear terms, the gaugino and the sfermion masses, which are calculated at the one-loop level. Electroweak Symmetry breaking (EWSB) occurs at the scale $M_{S U S Y}=\sqrt{\tilde{m}_{t_{1}} \tilde{m}_{t_{2}}}$ (where the one-loop contributions to the scalar potential are less relevant). At this scale we incorporate the SUSY threshold corrections to $m_{b}, m_{\tau}$ and $m_{t}$ by redefining the corresponding Yukawa couplings as done in ref. [47]. From $M_{S U S Y}$ to $M_{Z}$ we use the Standard Model renormalisation group equations. The running top mass is calculated iteratively, removing the Yukawa coupling $\lambda_{t}$, along with its derivative, from the remaining running from $m_{t}$ to $M_{Z}$.

Within this framework, for every set of input parameters, we perform an iterative integration of the RGE's (back and forth, from high to low energies). We define $M_{G U T}$ as the meeting point of the gauge couplings $\alpha_{1}$ and $\alpha_{2}$ and use this scale to find the unified value of the couplings, $\alpha_{U}$. The value $\alpha_{3}\left(M_{G U T}\right)$ is obtained from $\alpha_{3}\left(M_{M_{Z}}\right)$ (its 

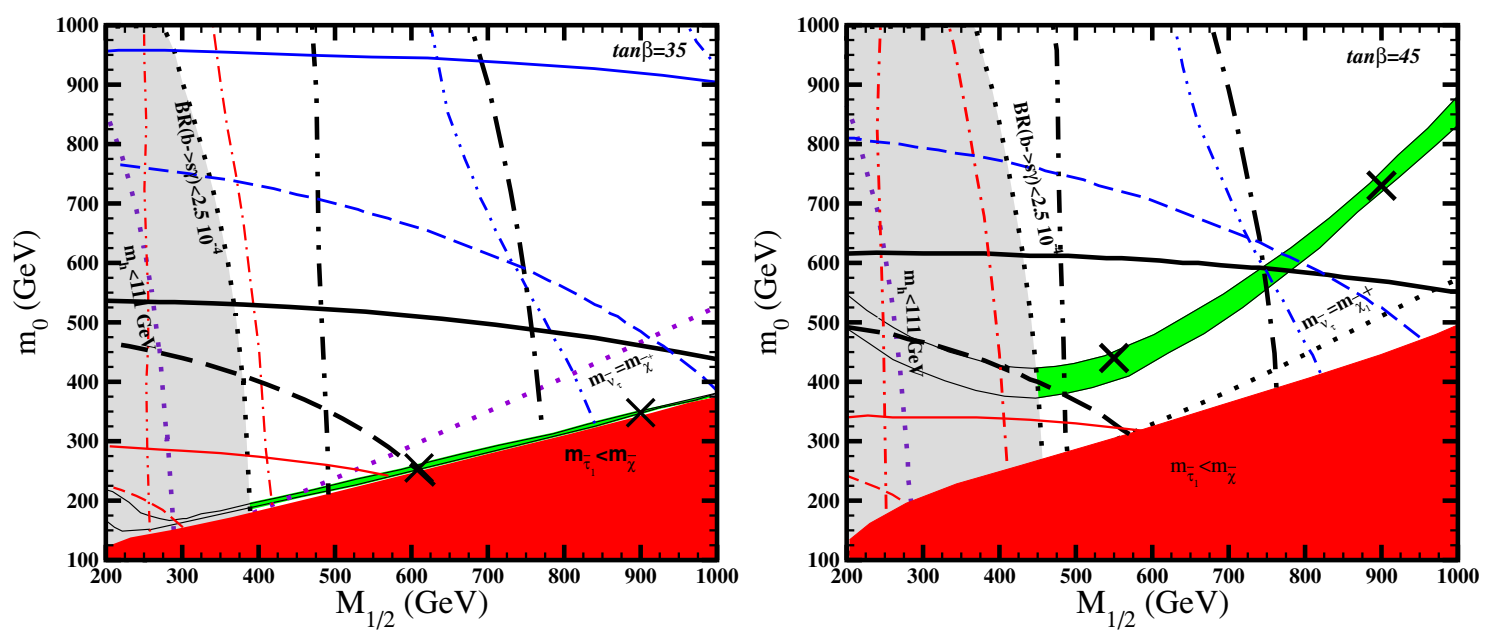

Figure 2. Contours of the cross sections $\sigma\left(e^{+} e^{-} \rightarrow \tilde{l}^{+} \tilde{l}^{-}\right)=1 \mathrm{fb}$ in the $\left(M_{1 / 2}, m_{0}\right)$ planes for $\tan \beta=35$ (left) and 45 (right) at three different energies, namely $\sqrt{s}=500 \mathrm{GeV}$ (red thin lines), $\sqrt{s}=1000 \mathrm{GeV}$ (black thick lines) and $\sqrt{s}=2000 \mathrm{GeV}$ (blue thin lines). The upper and lower nearhorizontal lines correspond to the following slepton pair production processes, respectively: $\tilde{\tau}_{1}^{+} \tilde{\tau}_{1}^{-}$ (solid) and $\tilde{\nu}_{\tau} \tilde{\nu}_{\tau}$ (dash). From left to right, the near-vertical lines correspond to $\sigma\left(e^{+} e^{-} \rightarrow \chi_{1}^{0} \chi_{2}^{0}\right)=$ $1 \mathrm{fb}$ (dot-dash) and $\sigma\left(e^{+} e^{-} \rightarrow \chi_{1}^{+} \chi_{2}^{-}\right)=1 \mathrm{fb}$ (double-dot-dash). In regions above the line where $m_{\tilde{\chi}_{1}^{+}}=m_{\tilde{\nu}_{\tau}}$ (purple dots), chargino-mediated processes are allowed. Representative contours of $\mathrm{BR}(b \rightarrow s \gamma)$ are indicated, and the regions with LSP relic density in the range preferred by WMAP are shaded green. The benchmark points selected for further study are indicated by crosses.

deviation from $\alpha_{U}$ being very small). The third generation Yukawa couplings are obtained by using the following set of values: $m_{\tau}\left(M_{Z}\right)=1.7463 \mathrm{GeV}$ (which takes into account the $\mathrm{SM}$ radiative corrections) and the top pole mass $m_{t}=172.6 \mathrm{GeV}$ [48]. For $m_{b}$ we take $m_{b}\left(M_{Z}\right)=2.92 \mathrm{GeV}$ which is the value where $m\left(m_{b}\right)=4.25 \mathrm{GeV}$ is mapped, taking $\alpha_{s}(M Z)=0.1172$. Note that the value of the bottom Yukawa coupling extracted from $m_{b}\left(M_{Z}\right)$ is very sensitive to the SUSY threshold corrections, especially at large $\tan \beta$; in our case we implement a complete one loop contributions as in ref. [49].

In figure 2 we present contours of the production cross sections for sparticles that are relevant for our study of LFV effects within the CMSSM, using $1 \mathrm{fb}$ as a reference cross-section value. We indicate in green the constraint on the parameter space derived from the WMAP-favoured CMSSM parameter space $\left(0.094<\Omega h^{2}<0.128\right.$ at $\left.3-\sigma\right)$ using MicrOMEGAS [50], and in grey the regions excluded by the constraints that $\mathrm{BR}(b \rightarrow s \gamma)$ $>2.5 \cdot 10^{-4}$ and $m_{h}>111 \mathrm{GeV}$ ( we assign an uncertainty of $\sim 3 \mathrm{GeV}$ to the theoretical calculation of $m_{h}$ ). We present contours for the production of $\tilde{\tau}_{1}^{+} \tilde{\tau}_{1}^{-}$calculated using CalcHEP [51]: the cross sections for producing other right sleptons would have similar contours, though at somewhat smaller values of $\left(M_{1 / 2}, m_{0}\right)$ because they are heavier than the $\tilde{\tau}_{1}$. The left slepton production cross sections are smaller, in general, and here we draw the $\sigma\left(\tilde{\nu}_{\tau} \tilde{\nu}_{\tau}\right)=1 \mathrm{fb}$ contour (the cross sections for all slepton pair-production processes are compared in figure 3). The relevant channels for LFV in cascade decays following pair production are also displayed in figure 2, along with the line $m_{\tilde{\chi}_{1}^{+}}=m_{\tilde{\nu}_{\tau}}$, above which chargino-mediated LFV processes are allowed. 
We would also like to note the following, regarding comparisons with known results: For $\tan \beta=30$ our results compare very well with the ones obtained using the SUSPECT code [52], as implemented in Micromegas [50]. At $\tan \beta=45$ we observe very small differences, due to the fact that we include all SUSY threshold corrections to $m_{b}$ and $m_{\tau}$ (from ref. [47]). Note that the resonances on the $\chi$ annihilation channels, relevant at large $\tan \beta$, are dependent on the value of $\lambda_{b}$, and therefore on the accuracy of the approximation in computing $m_{b}$. To quantify the difference, we note that our results for $\tan \beta=45$ are similar to the ones obtained with suspect for $\tan \beta=46$ and $m_{b}\left(m_{b}\right)=4.4 \mathrm{GeV}$.

Focusing initially on the lines corresponding to $\sqrt{s}=500 \mathrm{GeV}$, we see that on-shell production of left sleptons would occur mostly in the region excluded by $b \rightarrow s \gamma$. At this energy, therefore, we expect right slepton production to be more important. However, production of charged slepton pairs in the cascade decays of neutralinos is also possible, whilst chargino decays are disfavored. At $\sqrt{s}=1000 \mathrm{GeV}$, we see a significant part of the parameter space with left slepton pair production (below the dashed line) and with both left and right slepton production (below the solid line). The production of pairs of charged sleptons in chargino cascade decay is not possible in most of the WMAP favored area, since $m_{\tilde{\chi}_{1}^{+}}<m_{\tilde{\nu}_{\tau}}$. Finally, at $\sqrt{s}=2000 \mathrm{GeV}$ all the decay channels are present in the region of parameter space favoured by WMAP.

Based on this discussion, two benchmark points in the allowed regions for each of the choices $\tan \beta=35,45$ have been selected for subsequent study, namely:

(a35) $\tan \beta=35 m_{0}=255 \mathrm{GeV}, \quad \mathrm{M}_{1 / 2}=610 \mathrm{GeV}$,

(b35) $\tan \beta=35 m_{0}=345 \mathrm{GeV}, \quad \mathrm{M}_{1 / 2}=900 \mathrm{GeV}$,

(a45) $\tan \beta=45 m_{0}=440 \mathrm{GeV}, \quad \mathrm{M}_{1 / 2}=550 \mathrm{GeV}$,

(b45) $\tan \beta=45 m_{0}=730 \mathrm{GeV}, \quad \mathrm{M}_{1 / 2}=900 \mathrm{GeV}$

with $A_{0}=0$ in each case, resulting in the particle spectra shown in table 1 . We note that all these points lie beyond the regions of CMSSM parameter space excluded by 2010 LHC data [39-42]. In general, these points lead to more promising detection prospects at a LC at high energies: at $\sqrt{s}=500 \mathrm{GeV}$, only production through right sleptons is allowed.

We show in figure 3 the dependence of the flavour-conserving (FC) cross sections on the $\sqrt{s}$ of the collision for the benchmark points (3.1) with $\tan \beta=35$. The cross sections for the benchmarks with $\tan \beta=45$ are very similar. We can already get an idea of the LFV cross sections from the magnitudes of these FC cross sections, by looking at the approximate formulas (2.12) and (2.13), which assume that only 2-3 mixing is present. These provide an initial picture of the order of magnitude of LFV (up to $40 \%$ error), simply by convoluting the slepton flavour-violating decays with the FC cross sections. The latter can be extracted automatically, e.g., from CalcHEP [51], allowing one to explore quickly the relevant parameter space. If the mixing was indeed limited to the 2-3 sector, the accuracy would be larger, within $10 \%$ of the exact calculation discussed at a later stage.

As shown below, taking into account the full flavor mixing in the vertex $[11,27]$ leads to additional channels and an enhancement in the cross sections $\sigma\left(e^{+} e^{-} \rightarrow \tilde{\ell}_{i} \tilde{\ell}_{j}\right)$, also for the 


\begin{tabular}{|c|c|c|c|c|c|c|c|c|c|}
\hline & & & & & & & \\
\hline & & & Point & $m_{0}$ & $M_{1 / 2}$ & $\tan \beta$ & & & \\
\hline & & & a35 & 255 & 610 & 35 & & & \\
\hline & & & b35 & 345 & 900 & 35 & & & \\
\hline & & & a45 & 440 & 550 & 45 & & & \\
\hline & & & $\mathrm{b} 45$ & 730 & 900 & 45 & & & \\
\hline$M_{\tilde{\chi}_{1}^{+}}$ & $M_{\tilde{\chi}_{2}^{+}}$ & $M_{\tilde{\chi}_{1}^{0}}$ & 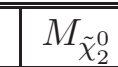 & $\bar{M}_{\tilde{\tau}_{1}}$ & $\bar{c} M_{\tilde{\tau}_{2}}$ & $\bar{~} \bar{M}_{\tilde{\ell}_{R}}$ & $M_{\tilde{\ell}_{L}}$ & $M_{\tilde{\nu}_{\tau}}$ & $M_{\tilde{\nu}_{e}}$ \\
\hline 480 & 741 & 255 & 480 & 261 & 477 & 342 & 478 & 454 & 472 \\
\hline 720 & 1034 & 383 & 720 & 385 & 670 & 478 & 683 & 655 & 679 \\
\hline 432 & 671 & 229 & 433 & 338 & 543 & 485 & 571 & 519 & 566 \\
\hline 725 & 1015 & 385 & 725 & 590 & 872 & 801 & 936 & 858 & 933 \\
\hline
\end{tabular}

Table 1. Relevant sparticle masses (in $\mathrm{GeV}$ ) for the selected benchmark points. The selected points all have $A_{0}=0$.

case of mixing in the LL sector, despite the fact that the mixing with the first generation is very constrained by the recent MEG bound $\operatorname{BR}(\mu \rightarrow e \gamma)<2.4 \cdot 10^{-12}[53]$.

The cross sections $e^{+} e^{-} \rightarrow \tilde{\ell}_{i}^{-} \tilde{\ell}_{i}^{+}, i=L, 1, R, 2$, which are relevant for calculating $e^{+} e^{-} \rightarrow \tilde{\ell}_{i}^{-} \tilde{\ell}_{j}^{+} \rightarrow \tau^{ \pm} \mu^{\mp} \tilde{\chi}_{1}^{0} \tilde{\chi}_{1}^{0}$, are displayed on the upper panels of figure 3 . We see that the production rates for left and right sleptons are comparable.

In the case of $e^{+} e^{-} \rightarrow \tilde{\nu}_{i} \tilde{\nu}_{j}^{c} \rightarrow \tau^{ \pm} \mu^{\mp} \tilde{\chi}_{1}^{+} \tilde{\chi}_{1}^{-}$, figure 3 indicates that the production rate for sneutrinos can be larger than that of charged sleptons. However, i) even if $e^{+} e^{-} \rightarrow \tilde{\nu}_{i} \tilde{\nu}_{j}^{c}$ is large, the decay $\tilde{\nu}_{i} \rightarrow l \tilde{\chi}_{1}^{-}$is allowed only in a restricted area of the parameter space, as we can see in figure 2 , and ii) the advantage of producing the $\tilde{\chi}_{1}^{ \pm}$is not so important in this case, because the decay $\tilde{\chi}_{1}^{ \pm} \rightarrow \chi+q_{u} q_{d}$ is not allowed in the range of $\tan \beta$ we favour (we recall that in the CMSSM the NLSP is usually a stau).

Finally, we comment on the cascade slepton production processes of eq. (2.9). These cases are not so promising within the CMSSM, but the analysis is simple, using the flavourconserving cross sections for charginos and neutralinos and multiplying by the LFV branching ratio. The LFV signal arises from the decay of the $\chi_{2}^{0}$ or $\chi_{2}^{+}$, which are produced at lower rates than the sleptons. The LFV decay of the $\chi_{2}^{0}$ has been already studied in the context of the LHC in [37]. Here, in agreement with [37], we find that mixing in the RR sector may lead to larger signals. However, the resulting cross sections are smaller than those mediated by sleptons. Also, the channel with $\chi_{2}^{+}$is not so relevant, not only because the production of charginos is lower than that of neutralinos (as can be seen in figures 3), but also because it is only sensitive to LL mixing in points where $m_{\tilde{\chi}_{1}^{+}}<m_{\tilde{\nu}_{\tau}}$.

Other production channels arising from $\tilde{\chi}_{2}^{ \pm} \tilde{\chi}_{2}^{\mp}$ and $\tilde{\chi}_{i}^{0} \tilde{\chi}_{j}^{0}$, where $i, j$ denote heavier neutralinos, are also open at higher energies and can contribute to the LFV signals. However, at the points we consider their production is lower than the one we study in detail and do not give any additional insight. 

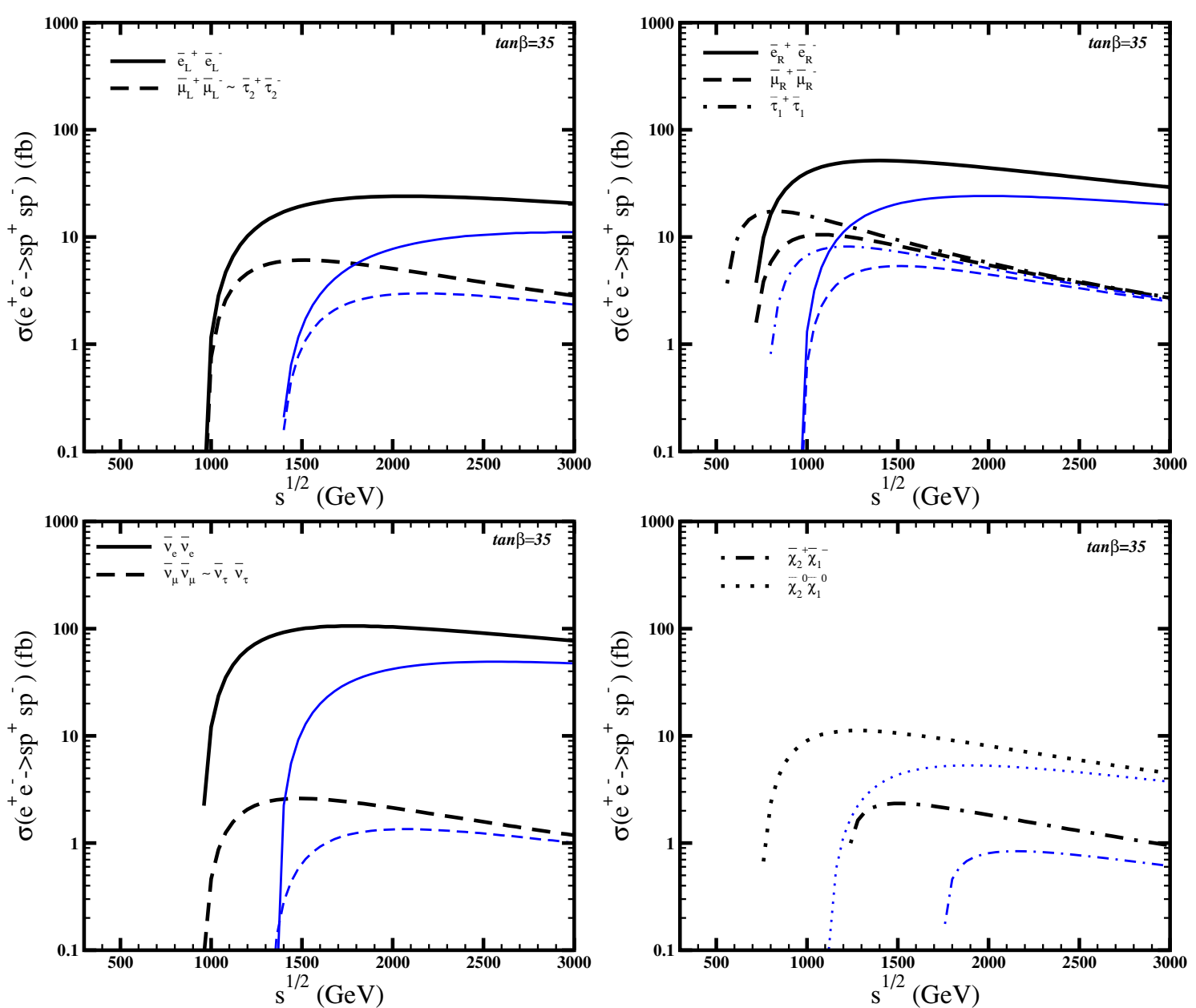

Figure 3. Energy dependence of the cross sections for sparticle production in the channels (2.8) and (2.9). The black thick (blue thin) lines correspond to point a35 (b35) in (3.1). The upper left (right) panels display left (right) charged slepton production, and the lower left (right) panels display sneutrino (gaugino) production.

\section{Opportunities for LFV observation at the LC}

LFV effects are severely constrained by decays of the form $\operatorname{BR}\left(\ell_{i} \rightarrow \ell_{j} \gamma\right)$, which limit significantly the allowed parameter space, with respect to both the mixing terms $\delta_{\mathrm{LL}, \mathrm{RR}}$ and the values of the soft supersymmetry-breaking masses. These limit the range of parameters in which we can get observable cross sections without violating the LFV bounds. In general, we may expect that LFV decays are progressively suppressed for a heavier sparticle spectrum at fixed values of $\delta_{\mathrm{LL}, \mathrm{RR}}$, and hence that one may get observable effects only for larger values of $\delta_{\mathrm{LL}, \mathrm{RR}}$. However, since the LC gives direct access to energies that are relatively high, the suppression induced by a heavier spectrum may be smaller than those in rare LFV decays and $\mu-e$ conversion. It is natural, therefore, to ask the following questions. (i) For a heavy sparticle spectrum, how small are the $\delta_{\mathrm{LL}, \mathrm{RR}}$ that are accessible 

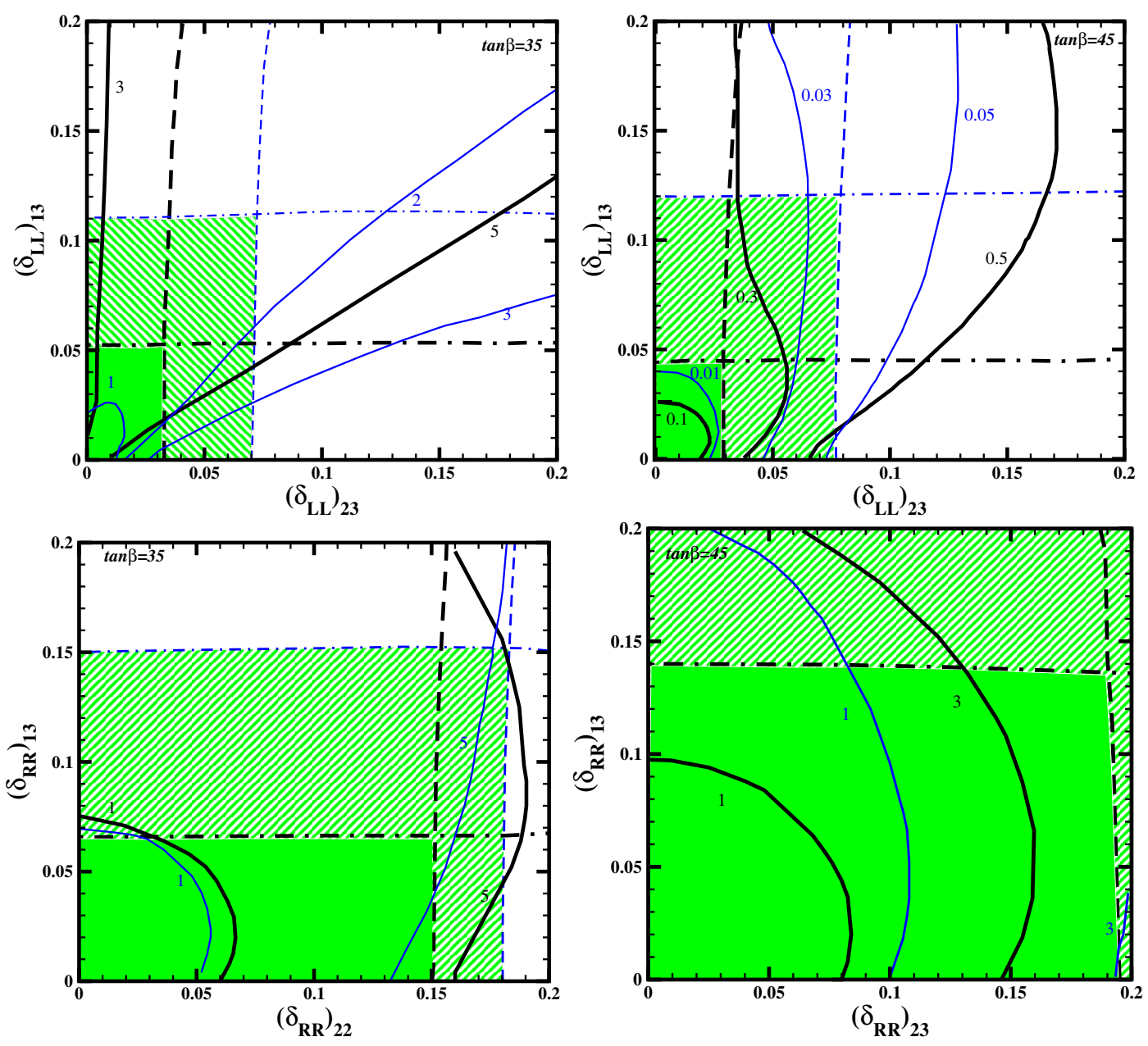

Figure 4. Constraints on the magnitudes of the mixing parameters and possible LFV effects for points with $\tan \beta=35$ (left panels) and $\tan \beta=45$ (right panels). The shaded areas are those allowed by current limits on $\operatorname{BR}(\tau \rightarrow e \gamma$ ) (dot-dash line) and $\operatorname{BR}(\tau \rightarrow \mu \gamma)$ (dash line) using a35, $a 45$ as reference points (thick lines bounding the solid shaded areas) and , $b 35, b 45$ (thin blue lines bounding the ruled shaded areas). The solid lines are contours of $\sigma\left(e^{+} e^{-} \rightarrow \tau^{ \pm} \mu^{\mp}+2 \chi^{0}\right)$ in fb for $\sqrt{s}=2000 \mathrm{GeV}$.

before the cross sections become too small to be observable? (ii) For a light sparticle spectrum, what are the minimum values of $\delta_{\mathrm{LL}, \mathrm{RR}}$ that can be probed?

These issues are addressed in figure 4 , where we display values of the flavour-violating parameters $\delta_{\mathrm{RR}}$ and $\delta_{\mathrm{LL}}$, showing lines of constant $\sigma\left(e^{+} e^{-} \rightarrow \tau^{ \pm} \mu^{\mp}+2 \chi^{0}\right)$ for $\sqrt{s}=2000$ $\mathrm{GeV} .{ }^{1}$ Using the bounds on $\tau \rightarrow \mu \gamma$ and $\tau \rightarrow \mu \gamma$ from [54], we display the allowed ranges in $\left(\left(\delta_{\mathrm{LL}}\right)_{23},\left(\delta_{\mathrm{LL}}\right)_{13}\right)$ planes (upper panels), and the corresponding mixing parameters in

\footnotetext{
${ }^{1}$ We choose $\sqrt{s}=2000 \mathrm{GeV}$ in figure 4 because, had we chosen $\sqrt{s}=1000 \mathrm{GeV}$, only the RR channels would lead to on-shell sleptons. Even for $\sqrt{s}=2000 \mathrm{GeV}$, we can see in figure 5 that point $b 45$ predicts very low cross sections for the LL case, due to the fact that the left sleptons are only marginally accessible, kinematically.
} 

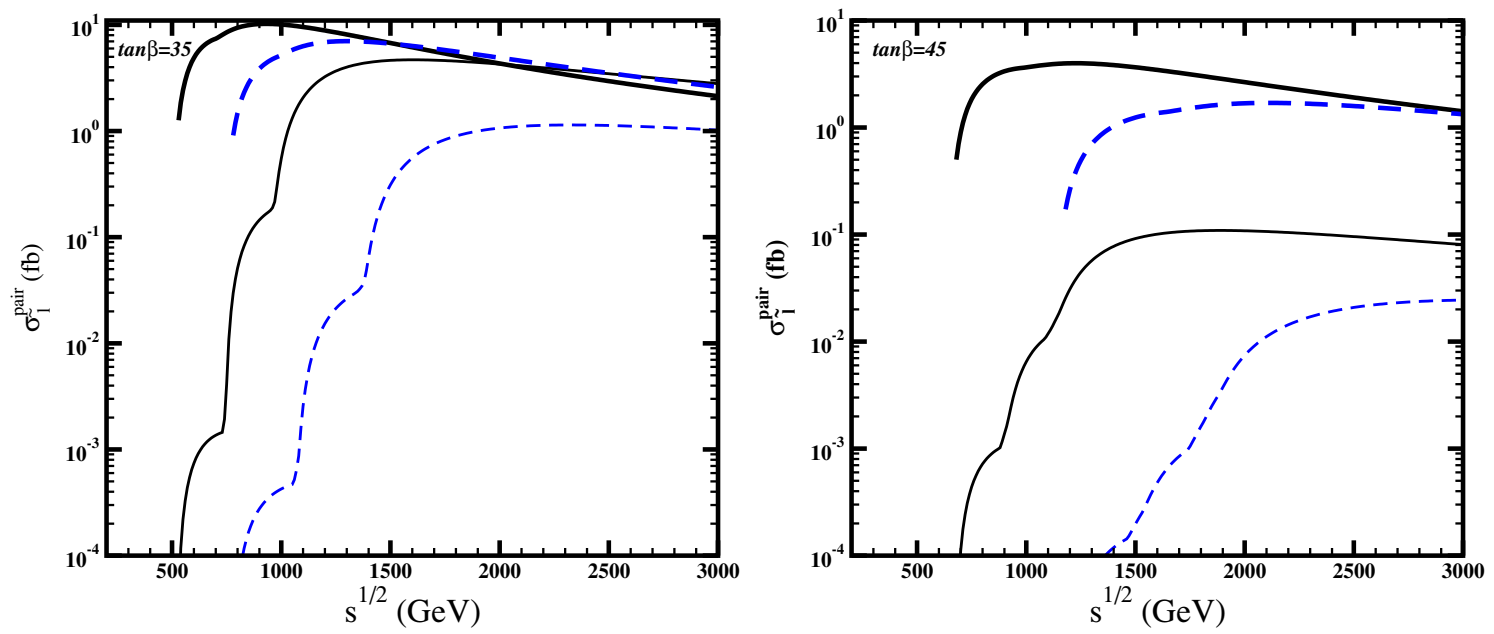

Figure 5. Values of $\sigma\left(e^{+} e^{-} \rightarrow \tilde{\ell}_{i}^{-} \tilde{\ell}_{j}^{+} \rightarrow \tau^{ \pm} \mu^{\mp}+2 \chi^{0}\right)$ vs $\sqrt{s}$ for the benchmark points with $\tan \beta=35$ (left) and $\tan \beta=45$ (right): solid (dashed) lines correspond to $a 35$ and $a 45$ ( $b 35$ and b45). Along the thick lines we assume the RR mixing of (4.2), and along the thin lines the LL mixing of (4.1).

the RR sector (lower panels). In this exploration, the values of $\left(\delta_{\mathrm{LL}, \mathrm{RR}}\right)_{12}$ were chosen to be proportional to those of $\left(\delta_{\mathrm{LL}, \mathrm{RR}}\right)_{13}$, so as to reduce the number of parameters. We have checked that the dependence of our results on these parameters are small, provided they are taken in a range such that the bound on $\operatorname{BR}(\mu \rightarrow e \gamma)$ is preserved. In addition, we note that the ratio $\left.\left(\delta_{\mathrm{LL}, \mathrm{RR}}\right)_{13}\left(\delta_{\mathrm{LL}, \mathrm{RR}}\right)_{12}\right)$ has a very small influence in $\tau \rightarrow \mu \gamma(\tau \rightarrow e \gamma)$.

As we see in figure 4 , the constraints from the LFV branching ratios result in horizontal and vertical lines in the $\delta_{\mathrm{LL}}$ and $\delta_{\mathrm{RR}}$ planes, corresponding to a box of allowed parameters that scales with the masses in the spectrum. On the other hand, the lines of constant LFV cross sections depend strongly on the kinematical properties of the chosen point as well as on the size of the mixing. In particular, we note the following:

- The shapes of the lines are quite different for each benchmark point, as was to be expected from the variations of the cross sections between the different benchmark points in the WMAP favoured area. For instance, for LL mixing, the differences between the predictions for the two points at $\tan \beta=45$ (upper right panel in figure 4) can be understood from the behaviours of the cross sections shown in figure 5 .

- Except for LL mixing at $\tan \beta=45$, we obtain LFV cross sections in the fb range at points of the parameter space where LFV decay branching ratios are below the present experimental bounds. This reflects the observation that passing to a heavier spectrum increases significantly the allowed size of the mixing parameters, due to the rapid decrease of the LFV branching ratios for heavy sparticle spectra.

- It is interesting to remark that at the LC mixing in the LL sector is observable without exceeding the bounds on $\ell_{i} \rightarrow \ell_{j} \gamma[53,54]$, due to the presence of additional 
channels as compared to the LHC. In the case of the LHC [37], mixing in the RR sector (which is less constrained but would require a departure from the CMSSM) had turned out to be the most promising avenue for discovering flavour violation. This is a clear distinction between the two colliders, and implies an advantage for the LC, in that its searches can be more directly connected with neutrino mass and mixing parameters.

The above results motivate a particular choice of parameters for a more detailed discussion of LFV cross sections. We assume the same values of the mixing parameters for both choices of $\tan \beta$, namely:

$$
\begin{aligned}
& \left(\delta_{\mathrm{LL}}\right)_{13}=0.02, \quad\left(\delta_{\mathrm{LL}}\right)_{23}=0.02 \\
& \left(\delta_{\mathrm{RR}}\right)_{13}=0.04, \quad\left(\delta_{\mathrm{RR}}\right)_{23}=0.15 .
\end{aligned}
$$

For completeness, we also introduce a small mixing between the first and second generation: $\left(\delta_{\mathrm{LL} / \mathrm{RR}}\right)_{12}=0.2 \cdot\left(\delta_{\mathrm{LL} / \mathrm{RR}}\right)_{13}$. The LFV decays into $\tau-\mu$ pairs are not heavily dependent on this parameter while, with this choice, the bound on $\operatorname{BR}(\mu \rightarrow e \gamma)$ does not over-constrain the parameter space.

LFV in the $\mu-e$ channel is generally suppressed in our scheme, unless $\delta_{\mathrm{LR}}$ mixing is introduced. In our analysis, however, we do not include such a mixing, in order to be able to discuss separately the LFV arising from each chiral sector, thereby avoiding the additional model dependence associated with choosing $\delta_{\mathrm{LR}} \neq 0$. In the case of large RR mixing, non-trivial interference of the RR sector with the LL sector would arise through LR mixing. The $\mu \rightarrow e \gamma$ bound, which could almost be ignored in selecting RR and LL mixing, would now play a very important role, forcing us to either a very small part of the parameter space or to very small values of $\left(\delta_{\mathrm{LR}}\right)$. To reach the bound on radiative decays with LR contributions alone we would need: $\left(\delta_{\mathrm{LR}}\right)_{12} \sim 10^{-3},\left(\delta_{\mathrm{LR}}\right)_{13} \sim 10^{-2}[55]$.

We also followed the conservative approach of keeping $A_{0}=0$, avoiding the introduction of other new LFV parameters. The main consequence for our analysis of allowing $A_{0} \neq 0$ would be to allow the chiral composition of the staus to vary, since the first and second generations have small Yukawa couplings. However, additional LFV could be introduced by assuming a non-trivial mixing matrix for the $A$ terms. In this case, chiral mixing would occur for all generations, restricting among other effects the size of the RR mixing. The decay $\mu \rightarrow e \gamma$ is the most sensitive to LFV $A$ terms, and establishing a correlation between $\mu$ and $\tau$ decays without having a specific model would be non-trivial. Nevertheless, since the bound on $\mathrm{BR}(\mu \rightarrow e \gamma)$ imposes a very severe restriction on $\left(M_{\mathrm{LR}}\right)_{12}$, even assumptions such as $\left(M_{\mathrm{LR}}\right)_{23} \sim\left(M_{\mathrm{LR}}\right)_{13} \sim 100\left(M_{\mathrm{LR}}\right)_{12}$ would not alter our main conclusions.

\section{Study of LFV at the LC}

The next step is to understand the dependence of the results on the available $\sqrt{s}$. Clearly, at the LC, higher values of $\sqrt{s}$ provide accessible cross sections for heavier sparticle spectra, a potential advantage of the LC over the LHC, where it is relatively difficult to reach large values of the scalar masses. The variation of $\sigma\left(e^{+} e^{-} \rightarrow \tau^{ \pm} \mu^{\mp}+2 \chi^{0}\right)$ with $\sqrt{s}$ is displayed 

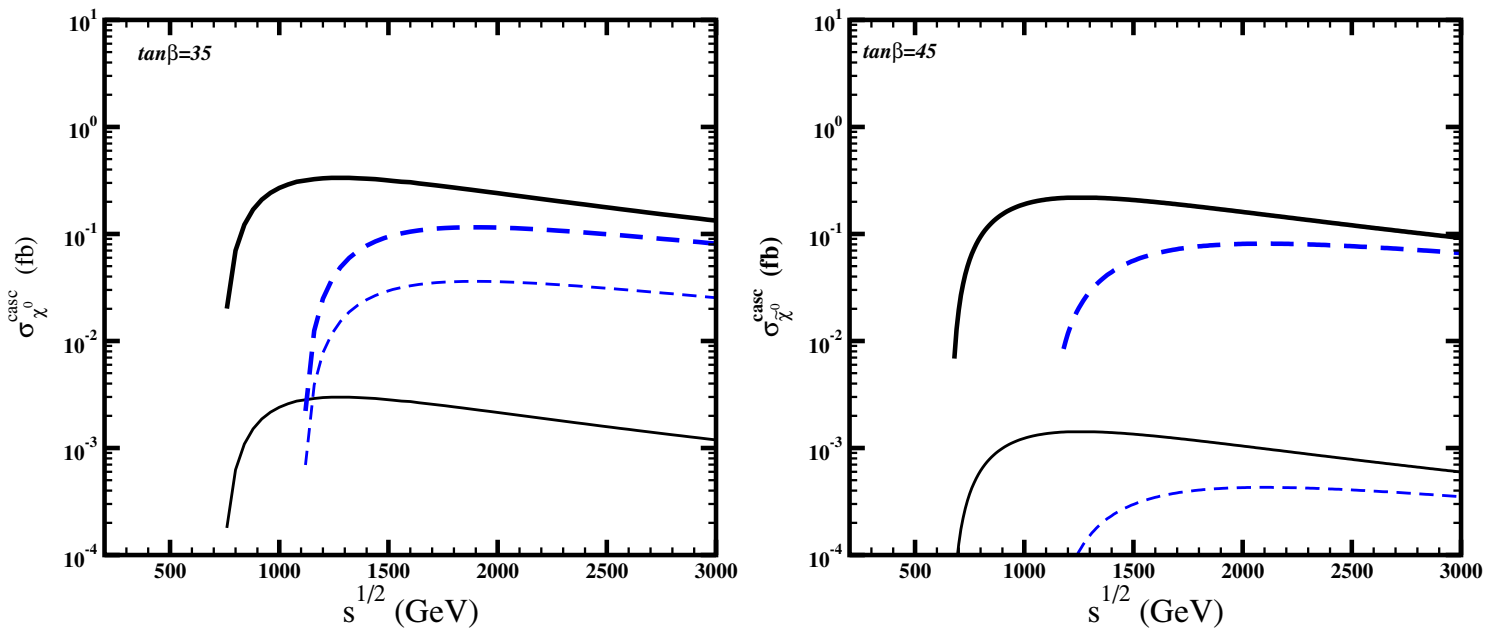

Figure 6. Same as in figure 5, but for cascade slepton production in the channel $\sigma\left(e^{+} e^{-} \rightarrow \chi_{1}^{0} \chi_{2}^{0} \rightarrow\right.$ $\left.\tau^{ \pm} \mu^{\mp}+2 \chi^{0}\right)$.

in figure 5 , where it is shown how, as $\sqrt{s}=2 P_{c m}$ increases, it becomes possible to produce on-shell sleptons with larger masses. We should underline once more that the effects due to the kinematics are as relevant as those from the LFV mixing parameters. The shapes of the lines can be understood as follows.

- The cross sections for slepton pair production show interesting dependence on $\sqrt{s}$, since additional channels arise from slepton mixing. We see in figure 5 steps in the left slepton production cross sections, which appear as the sleptons of the first two generations become kinematically accessible. The steps corresponding to lighter generations are less pronounced in the case of RR mixing than in the LL case.

- The overall cross sections for slepton production in cascade decays are independent of the LFV parameters, and LFV arises in the decays of the gauginos. Figures 6 and 7 display the cross sections for the pair production of $\tilde{\chi}_{2}^{0} \tilde{\chi}_{1}^{0}$ or $\tilde{\chi}_{2}^{+} \tilde{\chi}_{1}^{-}$multiplied by the LFV branching ratios for the decays of $\tilde{\chi}_{2}^{0}$ or $\tilde{\chi}_{2}^{+}$, computed as in [37]. This explains the simple shapes in this case.

- Distinguishing between pair and cascade slepton production is quite crucial, particularly since, as already mentioned, it is a fundamental difference between the LC and the LHC. Comparing the pair and cascade slepton production cross sections in figures 5 and 6 (where we chose similar scales to facilitate comparisons) we see the following: in slepton pair production, both LL and RR mixing effects are of the same order of magnitude; on the contrary, in the case of cascade slepton production, the channel with LL mixing has a cross section at least one order of magnitude less than that with RR mixing, offering a distinction between the two mixing scenarios.

- Channels with charginos in the final states could be promising when $m_{\tilde{\chi}_{1}^{+}}<m_{\tilde{\tau}_{1}}$, i.e., when the $\tilde{\chi}_{1}^{+}$is the NLSP. However, as already remarked, this is not the case of the 

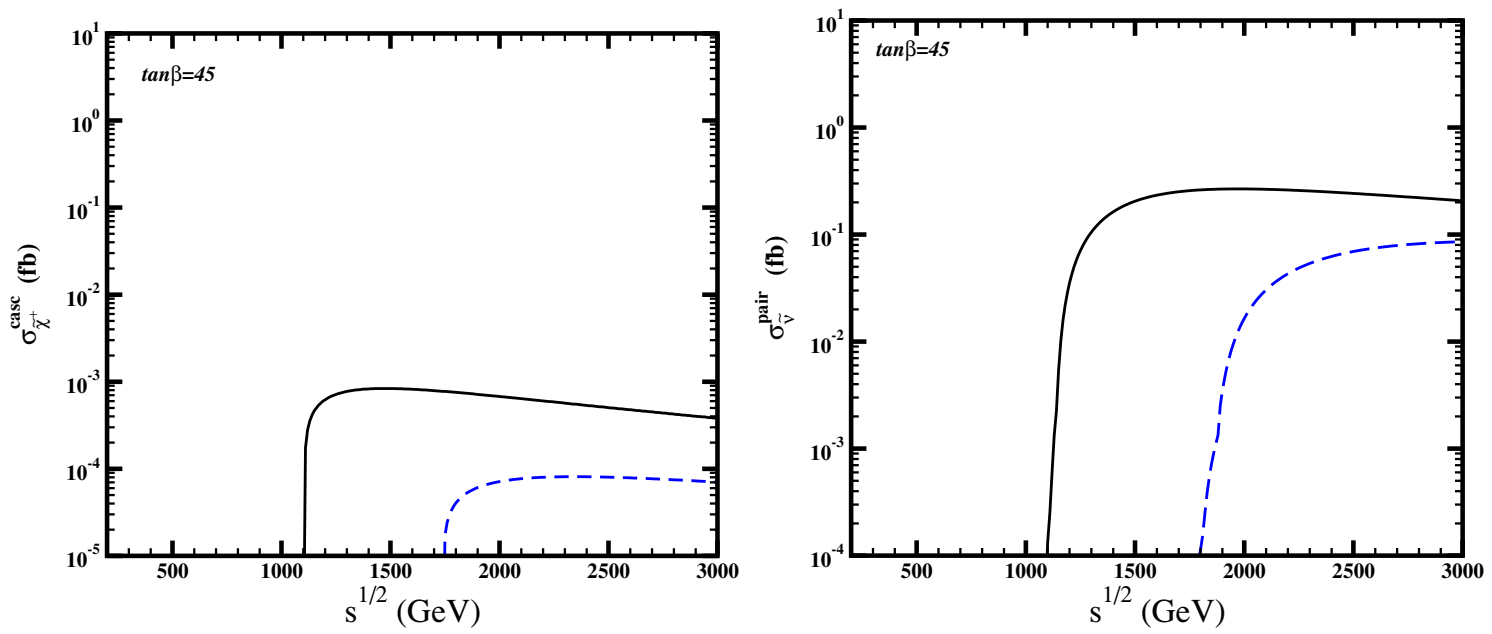

Figure 7. Left panel: same as in figure 5, but for cascade slepton production in the channel $\sigma\left(e^{+} e^{-} \rightarrow \chi_{1}^{ \pm} \chi_{2}^{\mp} \rightarrow \tau^{ \pm} \mu^{\mp}+2 \chi^{0}\right)$. Right panel: same as in figure 5 , but for pair production with sneutrino mediation $\sigma\left(e^{+} e^{-} \rightarrow \tilde{\nu}_{i} \tilde{\nu}_{j}^{c} \rightarrow \tau^{ \pm} \mu^{\mp}+\tilde{\chi}_{1}^{+} \tilde{\chi}_{1}^{-}\right)$. For $\tan \beta=35$ these channels are not allowed because $m_{\tilde{\nu}}<m_{\tilde{\chi}^{+}}$.

CMSSM. In general, the dominant chargino decay is $\tilde{\chi}_{1}^{+} \rightarrow \tilde{l} \nu$, thus we do not obtain additional information beyond that from simple slepton production and decay. This is true also for channels with the heavier neutralinos.

- Cascade slepton production mediated by charginos is possible only with mixing in the LL sector. Figure 7 (left panel) indicates that this channel could be relevant at $\sqrt{s}$ larger than in the neutralino mediated channels, and the cross sections would be of comparable magnitude in the LL mixing case. On-shell production requires $m_{\tilde{\nu}}>m_{\tilde{\chi}_{1}^{+}}$, which is not the case for the sample points with $\tan \beta=35$, as they lie in the coannihilation region of the WMAP allowed area. However, for $\tan \beta=45$ the WMAP region includes points with resonant annihilation of neutralinos where the condition $m_{\tilde{\nu}}>m_{\tilde{\chi}_{1}^{+}}$is satisfied.

- The expectations for sneutrino pair production and subsequent chargino production are shown in the right panel of figure 7. Relaxing the assumptions of the CMSSM can change the kinematics of $e^{+} e^{-} \rightarrow \tilde{\chi}_{1}^{ \pm} \tilde{\chi}_{2}^{\mp}$, as well as the mass ratio of $\tilde{\chi}_{2}^{+}$versus $\tilde{\nu}$ that determines the decay $\tilde{\chi}_{2}^{ \pm} \rightarrow \tilde{\chi}_{1}^{\mp} \tau^{ \pm} \mu^{\mp}$. The branching ratios of the $\tilde{\chi}_{1}^{ \pm}$decays to $\nu_{\tau} \tilde{\tau}_{1}$ are $80 \%$ and $65 \%$ at points $a 45$ and $b 45$ respectively, with a subsequent $\sim 100 \%$ decay of the stau into a $\tau$ and a $\chi_{1}^{0}$.

Cross sections for $e^{+} e^{-} \rightarrow \tau^{ \pm} \mu^{\mp}+2 \chi^{0}$ above $1 \mathrm{fb}$ can be reached in the areas of parameters shown in figure 8 for energies below those indicated. The large areas between the thick solid red, black and blue lines, on the one hand, and the shaded regions that are excluded by the indicated present experimental constraints, on the other hand, demonstrate 

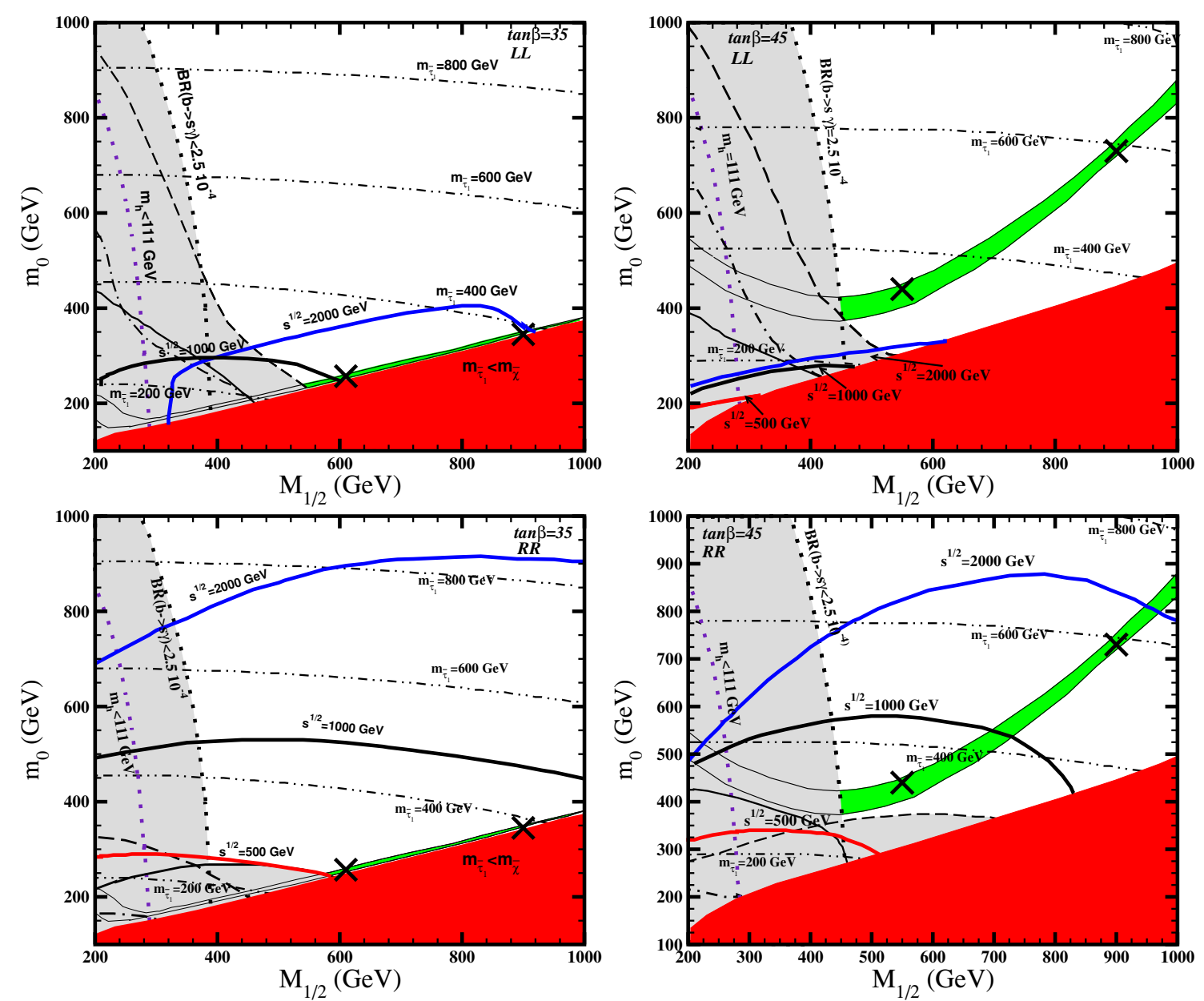

Figure 8. The solid red (black) (blue) lines are contours where $\sigma\left(e^{+} e^{-} \rightarrow \tau^{ \pm} \mu^{\mp}+2 \chi^{0}\right)=1 \mathrm{fb}$ at $\sqrt{s}=500,1000,2000 \mathrm{GeV}$, assuming the left mixing parameters (4.1) in the upper panels and the right mixing (4.2) in the lower panels. The left panels are for for $\tan \beta=35$ and the right panels for $\tan \beta=45$, assuming $A_{0}=0$ in both cases. Each panel also shows the areas excluded by current bounds on $\operatorname{BR}(\mu \rightarrow e \gamma)$ (thin solid line), $\operatorname{BR}(\tau \rightarrow e \gamma)$ (thin dash line), $\operatorname{BR}(\tau \rightarrow \mu \gamma)$ (thin dot-dash line). The areas excluded by $\mathrm{BR}(b \rightarrow s \gamma)$ and the LEP Higgs search are also displayed, and the green area denotes the WMAP favored region. We see that there are ample opportunities for LFV discovery and measurement at the LC, and the benchmark points chosen for further studies are indicated by crosses.

that there are ample opportunities for LFV discovery and measurement at the LC. These opportunities are exemplified by the benchmark points marked by crosses in figure 8 .

In figure 9 we present the maximum values of the cross sections in the areas allowed by all the current constraints. The shaded areas show the possible ranges of $\sigma\left(e^{+} e^{-} \rightarrow\right.$ $\tau^{ \pm} \mu^{\mp}+2 \chi^{0}$ ), and we note that points along the WMAP strips (solid lines) generally have high values of the cross sections. The dashed lines show the possible values of $\sigma\left(e^{+} e^{-} \rightarrow\right.$ $\left.\tau^{ \pm} e^{\mp}+2 \chi^{0}\right)$ along the WMAP strips, and we see that these cross sections may be of the same order of magnitude as those for $\mu-\tau$ pairs. $^{2}$

\footnotetext{
${ }^{2}$ We do not display values for $\sigma\left(e^{+} e^{-} \rightarrow \mu^{ \pm} e^{\mp}+2 \chi^{0}\right)$, because this would require a more precise analysis of mixings in the LR sector.
} 

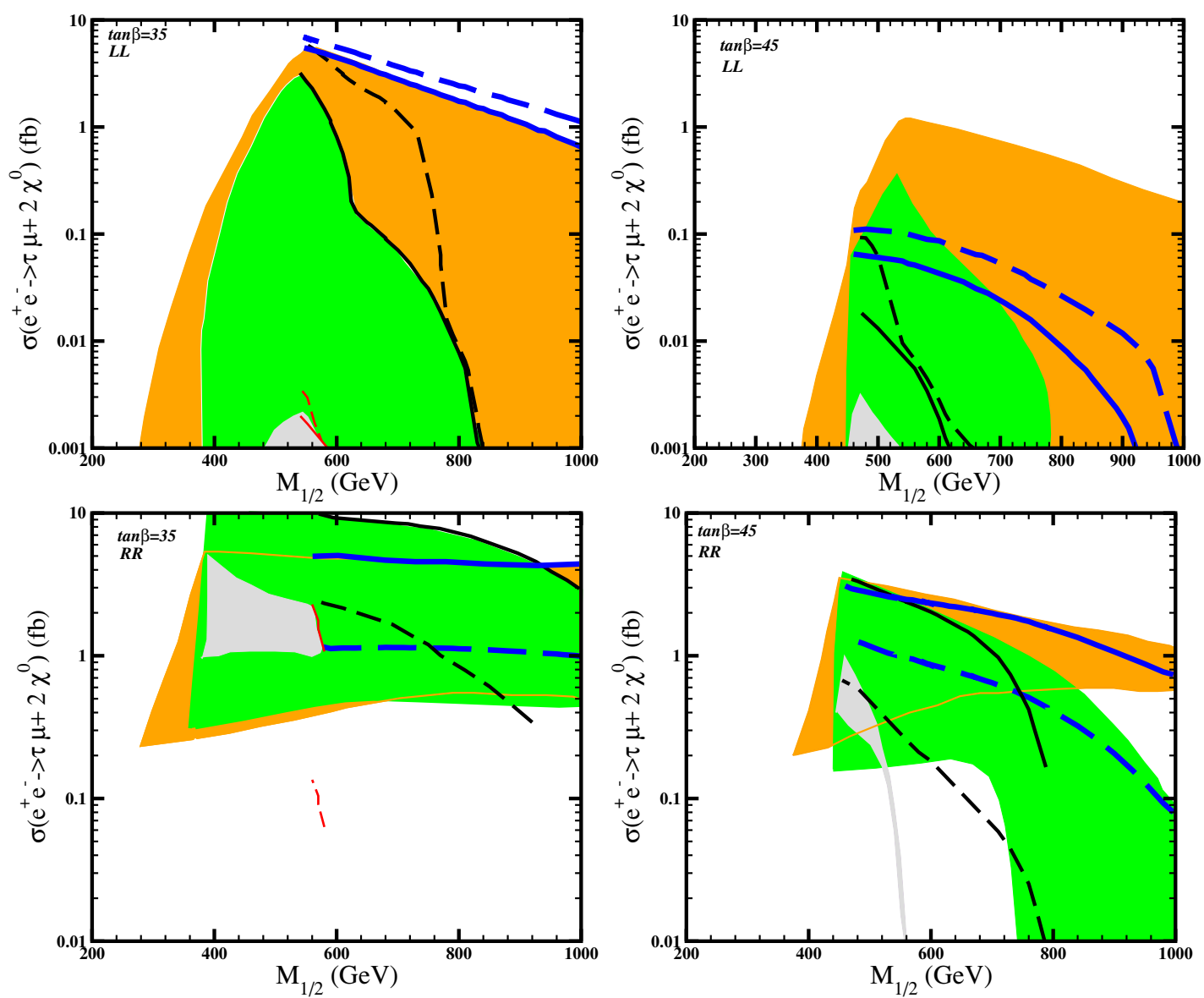

Figure 9. The shaded areas show the possible ranges of $\sigma\left(e^{+} e^{-} \rightarrow \tau^{ \pm} \mu^{\mp}+2 \chi^{0}\right)$ for $m_{0}<$ $1000 \mathrm{GeV}, \tan \beta=35$ (left panels) and $\tan \beta=45$ (right panels), assuming $A_{0}=0$ in both cases, with $\sqrt{s}$ fixed to $500 \mathrm{GeV}$ (grey), $1000 \mathrm{GeV}$ (green) and $2000 \mathrm{GeV}$ (orange). In the upper panels, we assume the left mixing parameters (4.1), whereas in the lower panels we assume the right mixing (4.2). The solid lines present the possible values for models along the center of the WMAP strips, with the lines corresponding to $\sqrt{s}=500,1000,2000 \mathrm{GeV}$ being progressively thicker. The corresponding predictions for $\sigma\left(e^{+} e^{-} \rightarrow \tau^{ \pm} e^{\mp}+2 \chi^{0}\right)$ are shown by the dashed lines.

In figure 10 we display the expectations for the production of LFV $\tau-\mu$ pairs in the cascade decays of eq. (2.9), which are suppressed compared to the pair production process of eq. (2.8). The production of LFV pairs from $\chi_{2}^{0}$ decays is analogous to the corresponding process at the LHC [37]. We observe that phenomenologically interesting values on the LL sector induce very low cross sections, whereas mixing in the RR sector may be more observable, as it enhances the expected rates.

The SM background can be as large as several tens of fb. However, it has been shown in ref. [30] that after imposing several cuts, it is possible to distinguish LFV signals at the level of $1 \mathrm{fb}$.

The SUSY background arises from flavor conserving channels like $e^{+} e^{-} \rightarrow \tilde{\nu}_{i} \overline{\tilde{\nu}}_{i}, e^{+} e^{-} \rightarrow$ $\tilde{l}_{i}^{+} \tilde{l}_{i}^{-}$and subsequent cascade decays that result in $\tau^{ \pm} \mu^{\mp}+\not$. This background is very 

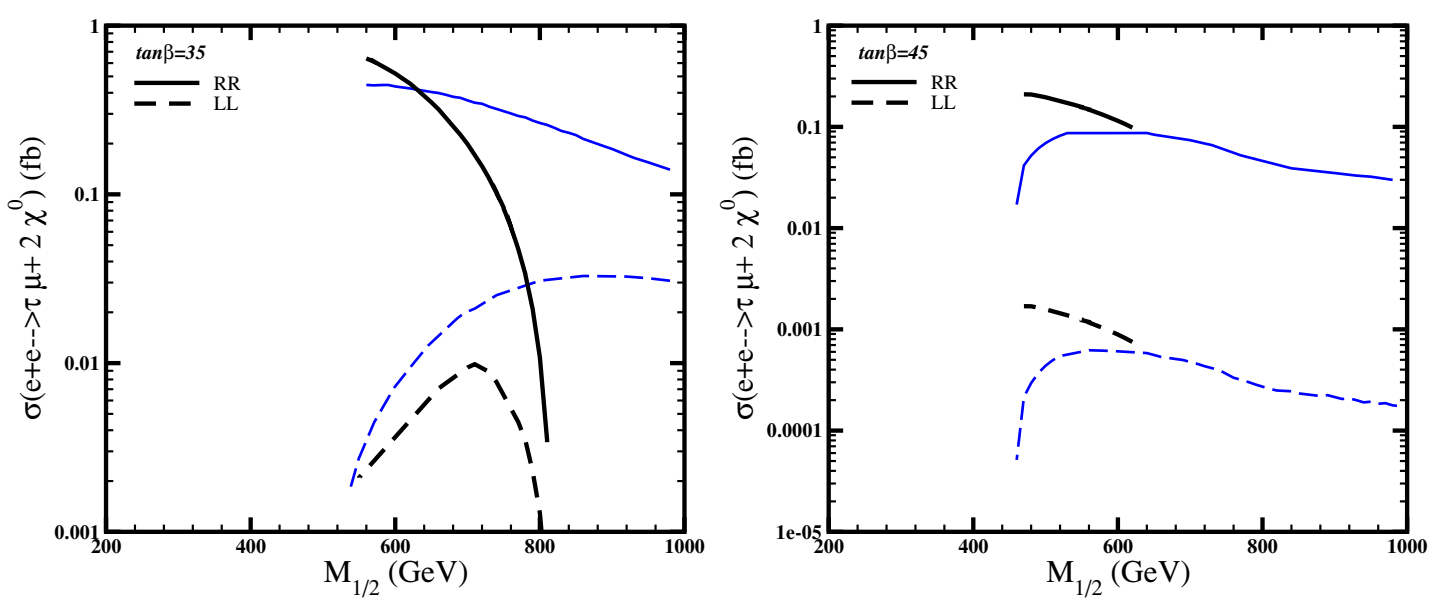

Figure 10. Predictions from $\chi_{2}^{0}$ cascade decays (2.9) along the WMAP strips for $\sqrt{s}=1000 \mathrm{GeV}$ (thick black lines) and $2000 \mathrm{GeV}$ (thin blue lines). These channels are not accessible at $\sqrt{s}=500$ $\mathrm{GeV}$. The solid (dashed) lines correspond to mixing in the RR (LL) sectors.

dependent on the SUSY spectrum and is much smaller than the SM one. In our case, the branching ratios for $e^{ \pm} \tau^{\mp}+\not E$ and $\mu^{ \pm} \tau^{\mp}+\not E$ arising from the production of $\tilde{e}_{L}^{+} \tilde{e}_{L}^{-}$ and $\tilde{\mu}_{L}^{+} \tilde{\mu}_{L}^{-}$are almost zero at the points with $\tan \beta=35$, and of about $10 \%$ at the points $a 45$ and $b 45$. Without considering any cut, this induces a background of less than $1 \mathrm{fb}$ in $\tau^{ \pm} \mu^{\mp}+\not$ at energies below $2 \mathrm{TeV}$. At $2 \mathrm{TeV}$, the background for $e^{ \pm} \tau^{\mp}+\not$ is bigger due to the higher production of $\tilde{e}_{L}$ and $\tilde{\nu}$ in $e^{ \pm} e^{\mp}$ collisions (while at $b 45$ it remains of the order of $1 \mathrm{fb}$, it reaches a value of $12 \mathrm{fb}$ at the point $a 45)$. Other channels like $e^{+} e^{-} \rightarrow \tilde{\chi}_{i}^{ \pm} \bar{\chi}_{j}^{\mp}$ and $e^{+} e^{-} \rightarrow \tilde{\chi}_{1}^{+} e^{-\overline{\nu_{\ell}}}$ produce backgrounds at the fb level even at $2 \mathrm{TeV}$.

\section{Conclusions}

Motivated by the challenging possibility of observable charged lepton flavour violation, which would provide a new window on flavour physics that illuminates novel aspects of supersymmetry, we have explored possible signatures at the Linear Collider (LC), combining phenomenological constraints with the cosmological considerations implied by WMAP. We have found that both direct slepton pair production and sleptons produced in cascade decays may provide interesting signals in the cosmologically-favoured region of the supersymmetric parametric space. Moreover, the LC could provide additional insights beyond those obtainable from the LHC, by virtue of its greater kinematic range for slepton production and its sensitivity to LL mixing as well as RR mixing.

Within this framework, we found the following.

- The LC enhances significantly the prospects of detecting LFV for heavy sparticle spectra, where flavour-violating rare decays and conversions are significantly suppressed. This allows probing an entirely different range of the flavour-violating parameters. 
- Unlike the LHC of ref. [37], at the LC we can have significant LFV within the CMSSM via LL mixing.

- The decay $e^{+} e^{-} \rightarrow \tau^{ \pm} \mu^{\mp}+2 \chi^{0}$ provides an optimal search channel in this respect. Channels where charginos play a significant role may require a departure towards theories where gaugino or Higgs unification is broken, in order to respect the conditions imposed by cosmology.

- Comparing direct slepton pair production with indirect slepton production through the cascade decays of heavier sparticles, we find that in the latter case, the cross sections induced by LL mixing are approximately one order of magnitude lower than in the RR mixing case. This is consistent with the results previously obtained for the LHC in ref. [37], and is due to the presence of additional channels in cascade pair production. These lead to an interesting scaling of the cross sections with the available energy, which is sensitive to slepton mixing parameters of the three generations.

It would be very interesting to further investigate the following issues.

- The cross sections expected in non-minimal extensions of the theory might not only enhance channels that in the current scheme are more suppressed, but could also enable a comparison of the allowed range of mixing parameters in different models.

- The expectations for models of massive neutrinos, in which quantum corrections provide a significant source of LFV in the LL channel, provide a potential link between LC observables and neutrino mass and mixing parameters.

- Detailed simulations of signals and backgrounds are desirable.

Overall, it seems that the LC provides an optimal environment for the study of LFV, whereas the LHC is limited to specific channels that have significant backgrounds. The fact that the LC opens up additional possibilities may prove significant for making the link between observable cross sections and flavour model building.

\section{Acknowledgments}

We would like to thank M. Cannoni and J. Rodríguez-Quintero for useful discussions. The work of J.E. is supported partly by the London Centre for Terauniverse Studies (LCTS), using funding from the European Research Council via the Advanced Investigator Grant 267352. S.L. thanks the CERN Theory Division for its kind hospitality. The work of M.E.G. is supported by the Spanish MCINN Consolider-Ingenio 2010 Programme under grant MultiDark CSD2009-00064, the project FPA2006-13825 and the project P07FQM02962 funded by "Junta de Andalucia".

Open Access. This article is distributed under the terms of the Creative Commons Attribution Noncommercial License which permits any noncommercial use, distribution, and reproduction in any medium, provided the original author(s) and source are credited. 


\section{References}

[1] Super-Kamiokande Collaboration collaboration, Y. Fukuda et al., Evidence for oscillation of atmospheric neutrinos, Phys. Rev. Lett. 81 (1998) 1562 [hep-ex/9807003] [INSPIRE].

[2] Super-Kamiokande Collaboration collaboration, Y. Fukuda et al., Constraints on neutrino oscillation parameters from the measurement of day night solar neutrino fluxes at super-kamiokande, Phys. Rev. Lett. 82 (1999) 1810 [hep-ex/9812009] [INSPIRE].

[3] Super-Kamiokande Collaboration collaboration, Y. Fukuda et al., Measurement of the solar neutrino energy spectrum using neutrino electron scattering,

Phys. Rev. Lett. 82 (1999) 2430 [hep-ex/9812011] [INSPIRE].

[4] SNO Collaboration collaboration, Q. Ahmad et al., Measurement of the rate of $\nu / e+d$ $\rightarrow p+p+e^{-}$interactions produced by B-8 solar neutrinos at the Sudbury neutrino observatory, Phys. Rev. Lett. 87 (2001) 071301 [nucl-ex/0106015] [INSPIRE].

[5] KamLAnd Collaboration collaboration, K. Eguchi et al., First results from KamLAND: evidence for reactor anti-neutrino disappearance, Phys. Rev. Lett. 90 (2003) 021802 [hep-ex/0212021] [INSPIRE].

[6] KamLAnd Collaboration collaboration, T. Araki et al., Measurement of neutrino oscillation with KamLAND: evidence of spectral distortion, Phys. Rev. Lett. 94 (2005) 081801 [hep-ex/0406035] [INSPIRE].

[7] K2K Collaboration collaboration, M. Ahn et al., Indications of neutrino oscillation in a $250 \mathrm{~km}$ long baseline experiment, Phys. Rev. Lett. 90 (2003) 041801 [hep-ex/0212007] [INSPIRE].

[8] MinOs Collaboration collaboration, D. Michael et al., Observation of muon neutrino disappearance with the minos detectors and the NuMI neutrino beam, Phys. Rev. Lett. 97 (2006) 191801 [hep-ex/0607088] [INSPIRE].

[9] F. Borzumati and A. Masiero, Large muon and electron number violations in supergravity theories, Phys. Rev. Lett. 57 (1986) 961 [INSPIRE].

[10] Y. Kuno and Y. Okada, Muon decay and physics beyond the standard model, Rev. Mod. Phys. 73 (2001) 151 [hep-ph/9909265] [INSPIRE].

[11] J. Hisano, T. Moroi, K. Tobe and M. Yamaguchi, Lepton flavor violation via right-handed neutrino Yukawa couplings in supersymmetric standard model, Phys. Rev. D 53 (1996) 2442 [hep-ph/9510309] [INSPIRE].

[12] J. Hisano, D. Nomura and T. Yanagida, Atmospheric neutrino oscillation and large lepton flavor violation in the SUSY SU(5) GUT, Phys. Lett. B 437 (1998) 351 [hep-ph/9711348] [INSPIRE].

[13] W. Buchmüller, D. Delepine and F. Vissani, Neutrino mixing and the pattern of supersymmetry breaking, Phys. Lett. B 459 (1999) 171 [hep-ph/9904219] [InSPIRE].

[14] M. Gomez, G. Leontaris, S. Lola and J. Vergados, U(1) textures and lepton flavor violation, Phys. Rev. D 59 (1999) 116009 [hep-ph/9810291] [INSPIRE].

[15] J.R. Ellis, M. Gomez, G. Leontaris, S. Lola and D.V. Nanopoulos, Charged lepton flavor violation in the light of the super-kamiokande data, Eur. Phys. J. C 14 (2000) 319 [hep-ph/9911459] [INSPIRE]. 
[16] W. Buchmüller, D. Delepine and L.T. Handoko, Neutrino mixing and flavor changing processes, Nucl. Phys. B 576 (2000) 445 [hep-ph/9912317] [INSPIRE].

[17] J.L. Feng, Y. Nir and Y. Shadmi, Neutrino parameters, abelian flavor symmetries and charged lepton flavor violation, Phys. Rev. D 61 (2000) 113005 [hep-ph/9911370] [INSPIRE].

[18] J. Sato and K. Tobe, Neutrino masses and lepton flavor violation in supersymmetric models with lopsided Froggatt-Nielsen charges, Phys. Rev. D 63 (2001) 116010 [hep-ph/0012333] [INSPIRE].

[19] J. Hisano and K. Tobe, Neutrino masses, muon g-2 and lepton flavor violation in the supersymmetric seesaw model, Phys. Lett. B 510 (2001) 197 [hep-ph/0102315] [INSPIRE].

[20] S. Baek, T. Goto, Y. Okada and K.-i. Okumura, Muon anomalous magnetic moment, lepton flavor violation and flavor changing neutral current processes in SUSY GUT with right-handed neutrino, Phys. Rev. D 64 (2001) 095001 [hep-ph/0104146] [INSPIRE].

[21] S. Lavignac, I. Masina and C.A. Savoy, Tau $\rightarrow \mu \gamma$ and $\mu \rightarrow$ e gamma as probes of neutrino mass models, Phys. Lett. B 520 (2001) 269 [hep-ph/0106245] [INSPIRE].

[22] D. Carvalho, J.R. Ellis, M. Gomez and S. Lola, Charged lepton flavor violation in the CMSSM in view of the muon anomalous magnetic moment, Phys. Lett. B 515 (2001) 323 [hep-ph/0103256] [INSPIRE].

[23] T. Blazek and S. King, Muon anomalous magnetic moment and $\tau \rightarrow \mu \gamma$ in a realistic string inspired model of neutrino masses, Phys. Lett. B 518 (2001) 109 [hep-ph/0105005] [INSPIRE].

[24] M. Cannoni, S. Kolb and O. Panella, Lepton flavor violation in $e^{+-} e^{-} \rightarrow l^{+-} e^{-}(l=\mu, \tau)$ induced by $R$ conserving supersymmetry, Phys. Rev. D 68 (2003) 096002 [hep-ph/0306170] [INSPIRE].

[25] N. Arkani-Hamed, H.-C. Cheng, J.L. Feng and L.J. Hall, Probing lepton flavor violation at future colliders, Phys. Rev. Lett. 77 (1996) 1937 [hep-ph/9603431] [INSPIRE].

[26] N. Arkani-Hamed, J.L. Feng, L.J. Hall and H.-C. Cheng, CP violation from slepton oscillations at the LHC and NLC, Nucl. Phys. B 505 (1997) 3 [hep-ph/9704205] [InSPIRE].

[27] J. Hisano, M.M. Nojiri, Y. Shimizu and M. Tanaka, Lepton flavor violation in the left-handed slepton production at future lepton colliders, Phys. Rev. D 60 (1999) 055008 [hep-ph/9808410] [INSPIRE].

[28] M. Guchait, J. Kalinowski and P. Roy, Supersymmetric lepton flavor violation in a linear collider: the role of charginos, Eur. Phys. J. C 21 (2001) 163 [hep-ph/0103161] [InSPIRE].

[29] F. Deppisch, J. Kalinowski, H. Pas, A. Redelbach and R. Ruckl, Supersymmetric lepton flavor violation at the $L H C$ and $L C$, hep-ph/0401243 [INSPIRE].

[30] F. Deppisch, H. Pas, A. Redelbach, R. Ruckl and Y. Shimizu, The SUSY seesaw model and lepton flavor violation at a future electron positron linear collider, Phys. Rev. D 69 (2004) 054014 [hep-ph/0310053] [INSPIRE].

[31] N. Krasnikov, Search for flavor lepton number violation in slepton decays at LHC, JETP Lett. 65 (1997) 148 [hep-ph/9611282] [INSPIRE].

[32] S. Bityukov and N. Krasnikov, The search for charged sleptons and flavor lepton number violation at LHC (CMS), hep-ph/9806504 [INSPIRE]. 
[33] K. Agashe and M. Graesser, Signals of supersymmetric lepton flavor violation at the CERN LHC, Phys. Rev. D 61 (2000) 075008 [hep-ph/9904422] [INSPIRE].

[34] J. Hisano, R. Kitano and M.M. Nojiri, Slepton oscillation at large hadron collider, Phys. Rev. D 65 (2002) 116002 [hep-ph/0202129] [INSPIRE].

[35] I. Hinchliffe and F. Paige, Lepton flavor violation at the CERN LHC, Phys. Rev. D 63 (2001) 115006 [hep-ph/0010086] [inSPIRE].

[36] D. Carvalho, J.R. Ellis, M. Gomez, S. Lola and J. Romao, Tau flavor violation in sparticle decays at the LHC, Phys. Lett. B 618 (2005) 162 [hep-ph/0206148] [INSPIRE].

[37] E. Carquín, J. Ellis, M. Gómez, S. Lola and J. Rodríguez-Quintero, Search for tau flavour violation at the LHC, JHEP 05 (2009) 026 [arXiv: 0812.4243] [INSPIRE].

[38] LeP Working Group for Higgs boson searches, AlePh Collaboration, Delphi Collaboration, l3 Collaboration, OPAl Collaboration Collaboration, R. Barate et al., Search for the standard model Higgs boson at LEP, Phys. Lett. B 565 (2003) 61 [hep-ex/0306033] [INSPIRE].

[39] CMS Collaboration collaboration, V. Khachatryan et al., Search for supersymmetry in $P P$ collisions at $7 \mathrm{TeV}$ in events with jets and missing transverse energy, Phys. Lett. B 698 (2011) 196 [arXiv:1101.1628] [InSPIRE].

[40] ATlas Collaboration collaboration, G. Aad et al., Search for supersymmetry using final states with one lepton, jets and missing transverse momentum with the ATLAS detector in $\sqrt{s}=7 \mathrm{TeV}$ pp, Phys. Rev. Lett. 106 (2011) 131802 [arXiv:1102.2357] [INSPIRE].

[41] ATLAS Collaboration collaboration, G. Aad et al., Search for squarks and gluinos using final states with jets and missing transverse momentum with the ATLAS detector in $\sqrt{s}=7$ TeV proton-proton collisions, Phys. Lett. B 701 (2011) 186 [arXiv:1102.5290] [INSPIRE].

[42] O. Buchmueller, R. Cavanaugh, D. Colling, A. De Roeck, M. Dolan, et al., Supersymmetry and dark matter in light of LHC 2010 and XENON100 data, Eur. Phys. J. C 71 (2011) 1722 [arXiv:1106.2529] [INSPIRE].

[43] WMaP Collaboration collaboration, E. Komatsu et al., Seven-Year Wilkinson microwave anisotropy probe (WMAP) observations: cosmological interpretation, Astrophys. J. Suppl. 192 (2011) 18 [arXiv:1001.4538] [INSPIRE].

[44] J. Kalinowski, Supersymmetric lepton flavor violation at $e^{+} e^{-}$linear colliders, Acta Phys. Polon. B 32 (2001) 3755 [INSPIRE].

[45] M. Gomez, S. Lola, P. Naranjo and J. Rodriguez-Quintero, WMAP dark matter constraints on Yukawa unification with massive neutrinos, JHEP 04 (2009) 043 [arXiv:0901.4013] [INSPIRE].

[46] S.P. Martin and M.T. Vaughn, Two loop renormalization group equations for soft supersymmetry breaking couplings, Phys. Rev. D 50 (1994) 2282 [Erratum-ibid. D 78 (2008) 039903] [hep-ph/9311340] [INSPIRE].

[47] M.E. Gomez, T. Ibrahim, P. Nath and S. Skadhauge, WMAP dark matter constraints and Yukawa unification in SUGRA models with CP phases, Phys. Rev. D 72 (2005) 095008 [hep-ph/0506243] [INSPIRE]. 
[48] Tevatron Electroweak Working Group and CDF and D0 Collaboration collaboration, A combination of CDF and D0 results on the mass of the top quark, arXiv:0803.1683 [INSPIRE].

[49] M.E. Gomez, T. Ibrahim, P. Nath and S. Skadhauge, Sensitivity of supersymmetric dark matter to the B quark mass, Phys. Rev. D 70 (2004) 035014 [hep-ph/0404025] [INSPIRE].

[50] G. Bélanger et al., MicrOMEGAs, http://lapth.in2p3.fr/micromegas/.

[51] A. Pukhov et al., CalcHEP - a package for calculation of Feynman diagrams and integration over multi-particle phase space, http://theory. sinp.msu.ru/ pukhov/calchep.html.

[52] A. Djouadi, J.-L. Kneur and G. Moultaka, SuSpect: a Fortran code for the supersymmetric and Higgs particle spectrum in the MSSM, Comput. Phys. Commun. 176 (2007) 426 [hep-ph/0211331] [INSPIRE].

[53] MEG Collaboration collaboration, J. Adam et al., New limit on the lepton-flavour violating decay $\mu^{+} \rightarrow e^{+} \gamma$, arXiv:1107.5547 [INSPIRE].

[54] Particle Data Group collaboration, K. Nakamura et al., Review of particle physics, J. Phys. G G 37 (2010) 075021 [http://pdg.lbl.gov/] [INSPIRE].

[55] M. Ciuchini, A. Masiero, P. Paradisi, L. Silvestrini, S. Vempati, et al., Soft SUSY breaking grand unification: leptons versus quarks on the flavor playground,

Nucl. Phys. B 783 (2007) 112 [hep-ph/0702144] [INSPIRE]. 